\title{
Beam Tail Measurements using Wire Scanners at DESY
}

Suren Arutunian, Matthias Werner, and Kay Wittenburg

Citation: AIP Conference Proceedings 693, 129 (2003); doi: 10.1063/1.1638338

View online: https://doi.org/10.1063/1.1638338

View Table of Contents: http://aip.scitation.org/toc/apc/693/1

Published by the American Institute of Physics

\section{Articles you may be interested in}

Experience with wire scanners at SLC

AIP Conference Proceedings 281, 264 (2008); 10.1063/1.44346 


\title{
Beam Tail Measurements using Wire Scanners at DESY
}

\author{
Suren Arutunian ${ }^{1}$, Matthias Werner ${ }^{2}$, Kay Wittenburg ${ }^{2}$ \\ ${ }^{1}$ Yerevan Physics Institute, ${ }^{2} \mathrm{DESY}$
}

\begin{abstract}
Wire scanners are used usually to measure the profile of the core of the beam. Especially at high beam currents the wire has to flip very fast through the beam, otherwise it will burn This is not true for the tails of the beam where the wire can be moved very slowly (or even stay stationary). Two effects are described which can be used to determine the tail distribution of the beam with the help of a slow wire scan. First: Counting of the scattered beam (tail) particles by scintillation counters. The rate versus the wire position will give directly the particle distribution in the tail. Second: The temperature of the wire depends on the amount of beam particle interactions with the wire. A temperature increase leads to an elongation of the wire and a dramatic change of its tension. This can be measured by the change of the natural frequency of the wire. Both methods are very sensitive and have a large dynamic range. The devices will be described and first results will be presented.
\end{abstract}

\section{INTRODUCTION}

Wire scanners are used in many laboratories to measure very precisely the beam profile. Different readout philosophies are applied, depending on the specific beam parameters and geometries: detection of - secondary emission current, - bremsstrahlung, scattered beam particles, - shower particles. Most of the applications are limited in their signal to noise ratio and therefore in their dynamic range. However, carefully designed systems may reach a dynamic range of $10^{5}-10^{6}$ with a single scanner. Other authors used a combination of wire scanner and scraper to achieve a high dynamic range [1].

Two different readout systems for wire scanners with a dynamic range of up to $10^{8}$ will be discussed in this report. One method uses the readout of shower particles: During a fast scan in the beam core the signal of an adjacent scintillator is analyzed by an $\mathrm{ADC}$, during a very slow scan in the beam tails the signals from the scintillator are counted. The second method is based on a very sensitive measurement of the temperature of the wire, which is heated by the crossing beam particles. In the following sections the two ideas are described and first measurements are presented.

\section{TAIL SCANS USING COUNTING MODE}

The standard wire scanner used in nearly all accelerators at DESY is based on a design of the wire scanner developed at LEP and is described elsewhere [Ref. 2, 3]. At DESY either $7 \mu \mathrm{m}$ Carbon wires (proton beams) or $15 \mu \mathrm{m} \mathrm{SiC}$ wires (electron beams) are used. The driver and readout electronic developed at DESY [4] makes it possible to scan with a maximum speed of $1 \mathrm{~m} / \mathrm{s}$ through the beam core or to make very slow scans, or even to stop the wire at a certain position. Both modes have a position resolution of $1 \mu \mathrm{m}$. The signal from the adjacent fast scintillator/photomultiplier (NE104/XP2243B) is sampled bunch by bunch with a sampling rate of 10.5 $\mathrm{MHz}$ with a resolution of 14 bit. Additionally the electronics provide a counting option, which simply counts the number of applied fast TTL signals over a certain time period.

For the sensitive tail studies at the HERA proton accelerator a fast constant fraction discriminator was connected additionally to the photomultiplier. A fast NIM-to-TTL converter (Type: LeCroy 688AL) converted the NIM signals from the discriminator and generated the TTL signals with a length of about 50 ns. 
The threshold of the discriminator can be varied and was set to avoid early saturation (the maximum count rate is the bunch repetition rate of $10.4 \mathrm{MHz}$ at HERAp). The threshold defined the overall sensitivity of the counting system. The following picture 2 shows the spectrum of the deposited energy in the scintillator for $\mathrm{E}_{\mathrm{dep}}>1 \mathrm{keV}$ calculated by the Monte Carlo program GEANT 4 . The number of primary protons hitting the $8 \mu \mathrm{m}$ Carbon wire was $3.91 * 10^{7}$ while 496 make an energy deposition $E_{\text {dep }}>1 \mathrm{keV}$. The efficiency is then $496 / 3.91 * 10^{7}=1.27 \times 10^{-5}$; however, the efficiency can simply be reduced by increasing the threshold to higher values of $E_{\text {dep }}$. Figure 3 shows the expected count rates at different positions of the wire at an efficiency of $10^{-7}$. Wire positions between $2 \sigma$ and $6 \sigma$ can expect to provide sufficient count rates for tail measurements.

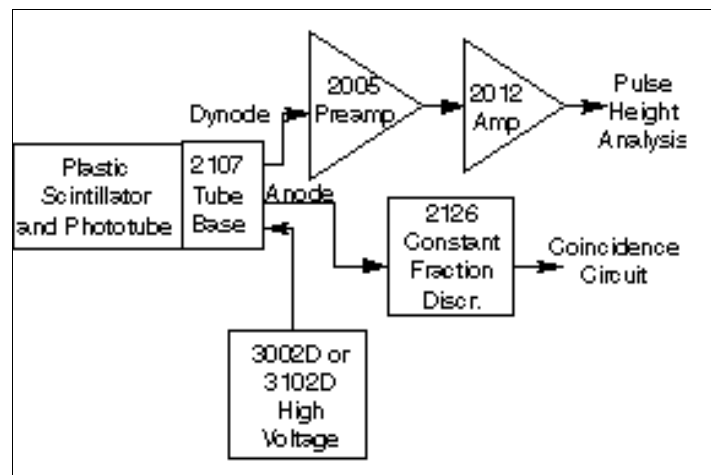

Figure 1.8: Plastic Scintillation Detector Electronics

FIGURE 1. Block diagram of the readout

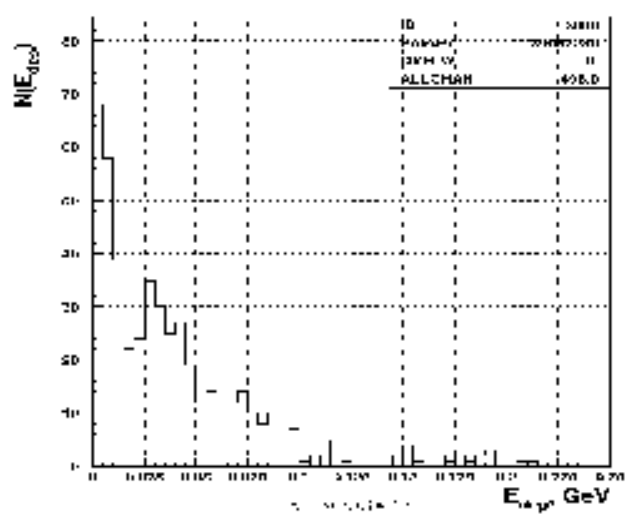

FIGURE 2. Spectrum of the deposited energy in the scintillator calculated by GEANT. The real geometry of the wire scanner setup in HERAp was used.

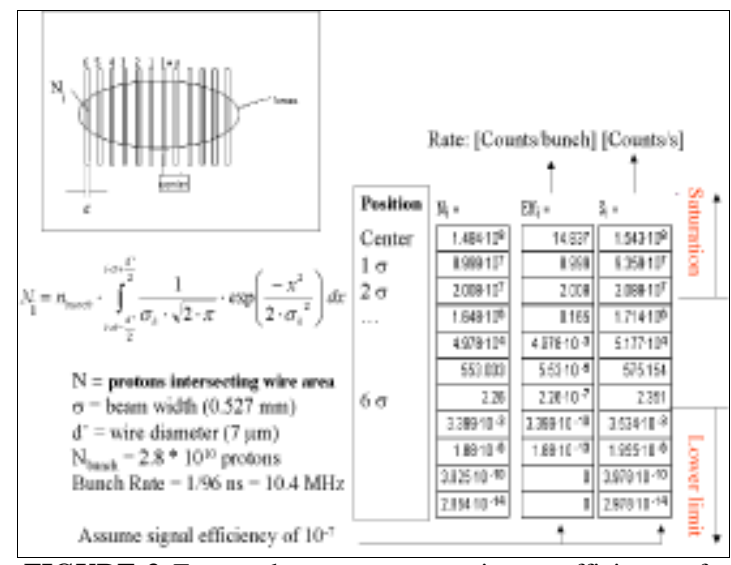

FIGURE 3. Expected count rate assuming an efficiency of $10^{-7}$ and a bunch rate of $10.4 \mathrm{MHz}$ at different wire positions.

\section{Measurements}

To achieve a large dynamic range, first a normal scan was made and the profile of the beam was stored and fitted by a gaussian. After that, a slow scan starting from the outside was made, in discrete steps. At each position the number of counts within $9 \mathrm{~s}$ was recorded. During this period the wire was kept at the same position. After the recording the wire was moved to the next position and the counts were recorded again. Since the fast and slow scan was made with exactly the same wire, the position information is identical. The overlap of the data from the fast and slow scan at around $2 \sigma$ (non saturated data) was used to normalize the counting data with the ADC data. In figures $4 a, b, c$ the profile from the fast scan together with the gaussian fit (4a) and two tail scans with different sensitivities together with the same fit are shown. These results show well the extended dynamic range as a result of the counting technique; it may reach up to $10^{8}$ ! Driving one scraper to about $6 \sigma$ from the beam core initiates the creation of the larger tails while the smaller tails were measured without any scraper.

\section{More measurements}

During the last years the HERA B experiment had take a lot of data. The internal wire targets of the experiment were brought to certain positions in the beam tails to achieve an adequate interaction rate. The experiment detected the interaction rate with an efficiency of about $50 \%$. A lot of tail studies were 


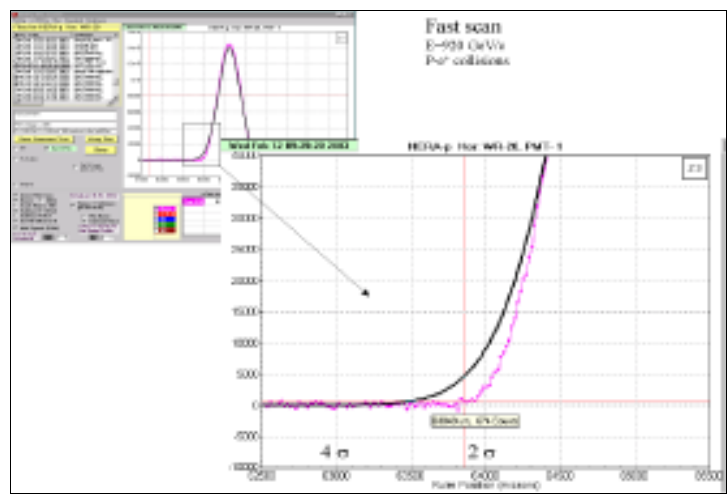

FIGURE 4A. Horizontal beam profile and a zoom on the tail distribution around $2 \sigma$ (fast scan). Note that this profile is the sum of 100 single bunch profiles. The black line shows a gaussian fit to the data.

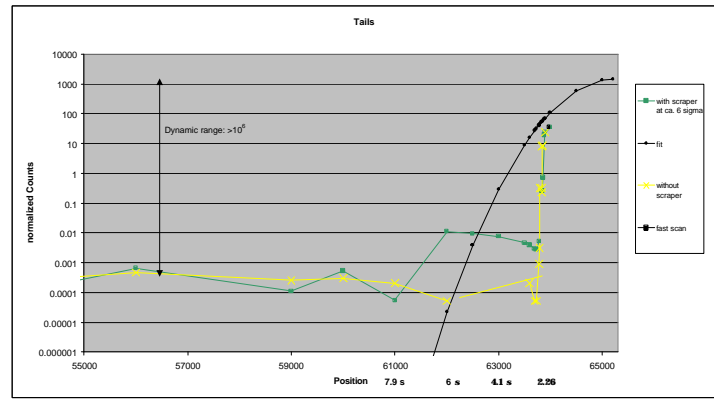

FIGURE 4B. Tail distributions around $6 \sigma$ measured by the counting method and normalized to the fast scan data of a single bunch.

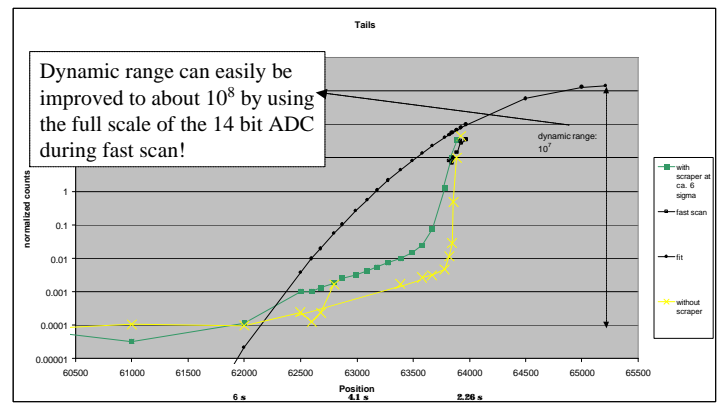

FIGURE 4C. Tail distributions around $6 \sigma$ measured by the counting method and normalized to the fast scan data of a single bunch. This measurement was done with a reduced threshold and therefore with an increased sensitivity.

done using the infrastructure of the experiment. Beside a much higher efficiency, the experiment provides also a high-resolution timing system. By gating the counts within successive time slots of $24 \mathrm{~ns}$ or $0.1 \mathrm{~ns}$, coasting beam between bunches and even the occupation of neighbor buckets $(500 \mathrm{MHz})$ were observable, respectively. These measurements are described in detail in [10]. However, this technique can be applied easily to the one-channel-readout of a wire scanner. Commercial available TDC or similar techniques have resolutions of much better than $1 \mathrm{~ns}$. Also photomultipliers with rise times of below $1 \mathrm{~ns}$ are available.

\section{Improvements}

Some improvements are planned to increase the resolution and the dynamic range: i) At high count rates the measurement should be linearised by applying Poisson statistic. ii) Applying a more reasonable fit to the tails of the fast measurement will improve the overlap conditions. iii) The lower count rate was limited by real beam losses to about $3 \mathrm{~Hz}$ at the higher threshold and about $40 \mathrm{~Hz}$ at the lower threshold. Applying a telescope technique with a triple coincidence can reduce this background. An example is given in [5]. vi) Applying of a time resolving counting technique, like the HERA-B technique.

\section{SCANNING EXPERIMENTS USING VIBRATING WIRE}

The principle of operation of the vibrating wire scanner is based on the dependence of the wire natural frequency $f_{0}$ on the beam intensity at the given location. The energy deposition of the beam particles in the wire causes heating of the wire. Hence the stretched wire temperature can be obtained by measuring its natural oscillations frequency by an autogenerator electronic circuit with a positive feedback loop. Initial experiments on profiling were done using laser beams $[6,7]$. The effective temperature precision was estimated to be about $10^{-4}$ degrees $C$ (without noise). Later experiments $[8,9]$ used the low intensity electron beam of the injector of the Yerevan synchrotron (bunches with RF of 2797.3 $\mathrm{MHz}$ with pulse duration of $2 \mu$ s and a repetition rate of $50 \mathrm{~Hz}$ ). A beryllium-bronze wire of $90 \mu \mathrm{m}$ diameter was used to scan the beam profile. Fig. 5 shows the result of the beam profile for the first scanning together with a normal distribution with $\sigma=1.48 \mathrm{~mm}$ and a beam central position at $30.87 \mathrm{~mm}$. The overall current of the beam was set to $\mathrm{I}_{0} \sim 10 \mathrm{nA}$. Only half of the beam could be scanned because of the short throw of the scanner.

\section{Calculations of "tail sensitivity"}

Predictions about the sensitivity according to the measurements were done in [9]. With a sensitivity of $0.01 \mathrm{~K}$ (assumed thermal sensitivity in the presence of 
noise), the estimations show that for a $90 \mu \mathrm{m}$ beryllium-bronze wire and $100 \mathrm{~mA}$ proton beam with $\sigma_{\mathrm{x}}=0.6 \mathrm{~cm}$ (PETRA conditions) the value $\mathrm{T}_{\mathrm{m}}$ of about $0.01 \mathrm{~K}$ (vibrating wire thermal sensitivity at presence of electromagnetic noises) is achieved at $x=5.7 \sigma$. The following estimation agrees well with this calculation: At $2 \sigma$ the wire was hit by $1.8 \cdot 10^{8} \mathrm{e} / \mathrm{s}$ (assuming $10 \mathrm{nA}$ beam current), showing the first increase of the temperature. For a $100 \mathrm{~mA}$ beam with a width of $\sigma=0.6 \mathrm{~cm}$ the same amount of hitting particles (and therefore the same increase if the temperature) will be reached at about $6.1 \sigma$.

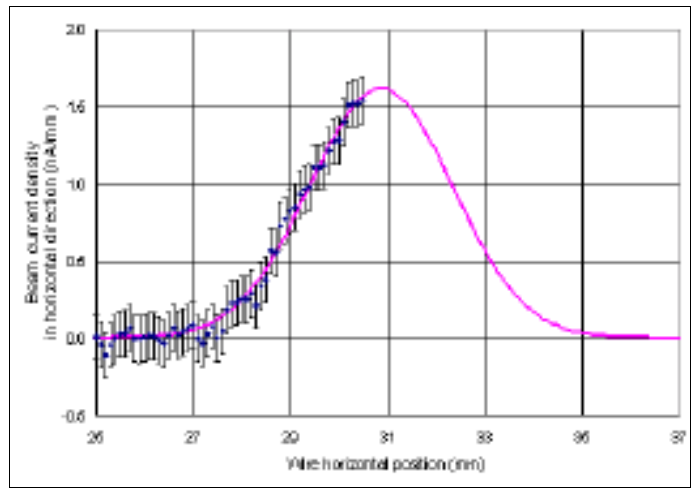

FIGURE 5. Reconstructed horizontal profile of an electron beam with a current of about $10 \mathrm{nA}$. The reconstruction was done after a detailed noise analysis; see $[8,9]$.

\section{Further studies}

One vibrating wire scanner is already installed in the PETRA proton ring at DESY. Further test are planned to determine: i) the influence of mechanical vibrations of the device on the measurement, ii) how well the theoretical predictions compare with measurements. The theory for the heating of the wire was developed mainly for DC beams. The authors expect, that it can be applied in the same way to bunched beams. Detailed studies with different bunch repetition rates are foreseen. iii) Higher order modes (HOM) might depose energy in the vibrating wire. At the SPS at CERN this effect was strong enough to damage the conducting Carbon wire [11]. The induced heating of the wire will disturb the vibration measurements. Test will to be done to damp the resonant modes using ferrites.

\section{ACKNOWLEDGMENTS}

A. Batalov (IHEP, Moscow) provided the Monte Carlo calculations. His results and discussions with him helped a lot for understanding the results.

\section{REFERENCES}

1. R. Macek, "Halo measurement diagnostic for the extracted beam in PSR", this workshop; D. Gilpatrick, "Wide Dynamic-Range Beam-Profile Instrumentation for a Beam-Halo Measurement: Description and Operation"; this workshop

2. B. Bouchet, et al., "Wire Scanners at LEP.", in CERNSL-91-20-DI. IEEE PAC San Francisco, CA, 6 - 9 May 1991, p. 1186-1188.

3. C. Bovet, A. Burns, F. Ferioli, Q. King, J. Koopman, J. Mann, H. Michel, R. Schmidt, L. Vos, "Experience with LEP Wire Scanners" in CERN-LEP-COMMISSIONINGNOTE-24, Apr 1990. 6pp.

4. M. Werner, K. Wittenburg, "A New Wire Scanner Controll Unit" in 5th European Workshop on Diagnostics and Beam Instrumentation, 13th -15th May, 2001, ESRF, Grenoble.

5. D. Gassner, "A Scanning Target Profile Monitor in the Slow Extracted Beam at the AGS" this workshop

6 Arutunian S.G., et al., "Vibrating Wire for Beam Profile Scanning" In: Phys. Rev. Spec. Top. Accel. Beams 2 (1999), pp.122801.

7 Arutunian S.G., et al.,. "Nonselective receiver of laser radiation on the basis of vibrating wire." - Proc. Conference Laser 2000 (November 2000, Ashtarak, Armenia)

8 Arutunian S.G. et al, "Vibrating wire scanner: first experimental result on the injector beam of Yerevan Synchrotron" Phys. Rev. ST Accel. Beams 6, pp. 042801 (2003)

9 Arutunian et al, "First Experimental Results and Improvements on Profile Measurements with the Vibrating Wire Scanner', in 6th European Workshop on Diagnostics and Beam Instrumentation 5 - 7 May, 2003, Mainz, Germany

10 S. Pratte, DESY, „Bestimmung der Wechselwirkungsrate des HERA-B Targets und Untersuchungen des Coasting Beam am HERA Protonen Ring“. Thesis, University Dortmund, June 2000.

11 F. Caspers, et al., "Cavity Mode Related Breaking of the SPS Wire Scanners and Loss Measurements of Wire Materials", in 6th European Workshop on Diagnostics and Beam Instrumentation 5 - 7 May, 2003, Mainz, Germany 